\title{
HOBS methods for enhancing resolution and sensitivity in small DNA oligonucleotide acid NMR studies
}

Josiah M. McKenna and John A. Parkinson

Supporting Information 


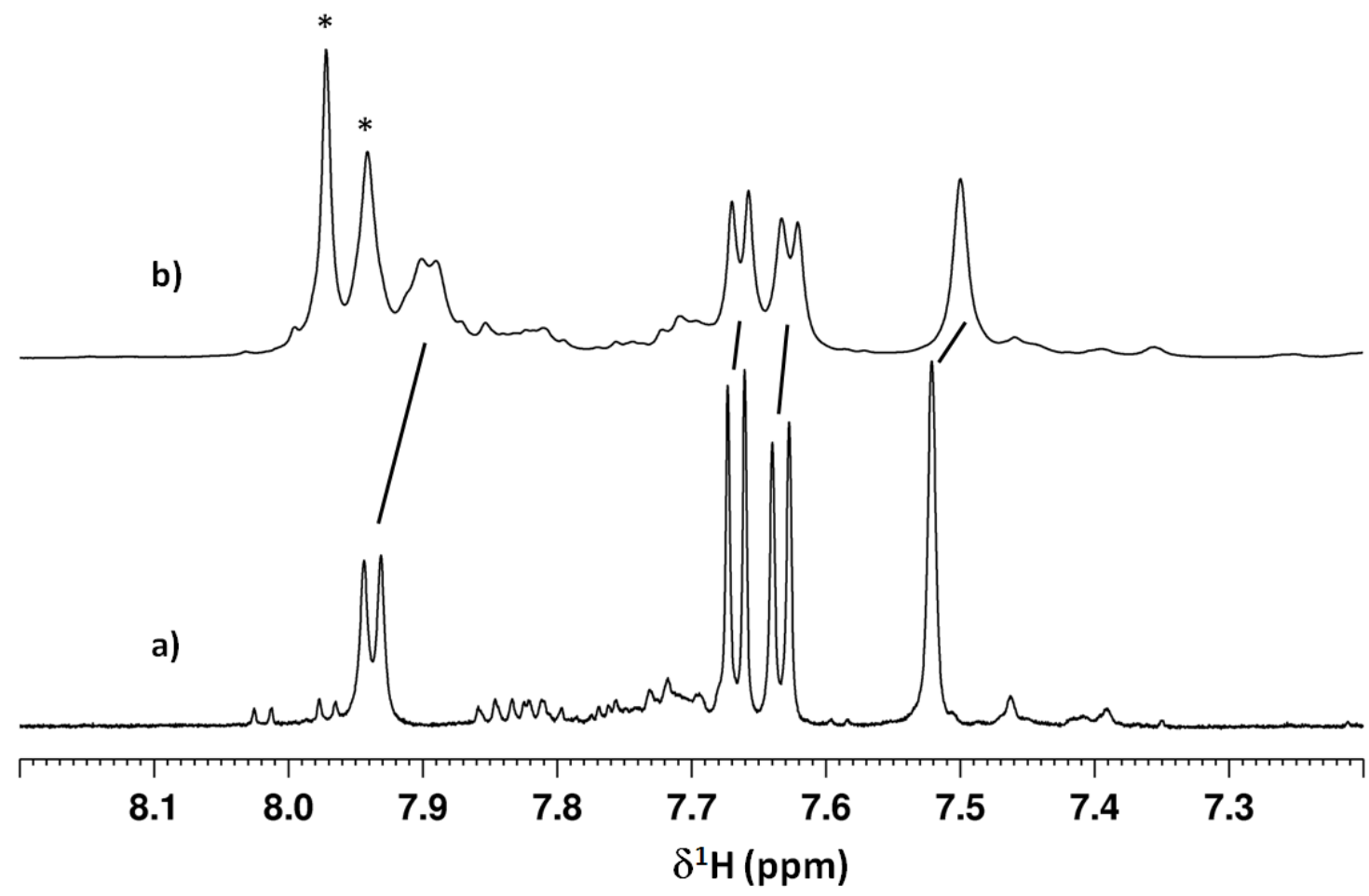

Figure S1: Aromatic region of the $600 \mathrm{MHz} 1 \mathrm{D}^{1} \mathrm{H}$ NMR spectrum of $\mathrm{d}(\mathrm{GCCTGC})$ in $\mathrm{D}_{2} \mathrm{O}$. a) Aged sample; b) freshly dissolved sample. Asterisks indicate $\mathrm{G} \mathrm{H} 8$ resonances which exchange with deuterium over extended periods of time. Note that sample concentrations differ in the two cases resulting in linewidth and small chemical shift differences for those proton resonances that are common to both spectra. 

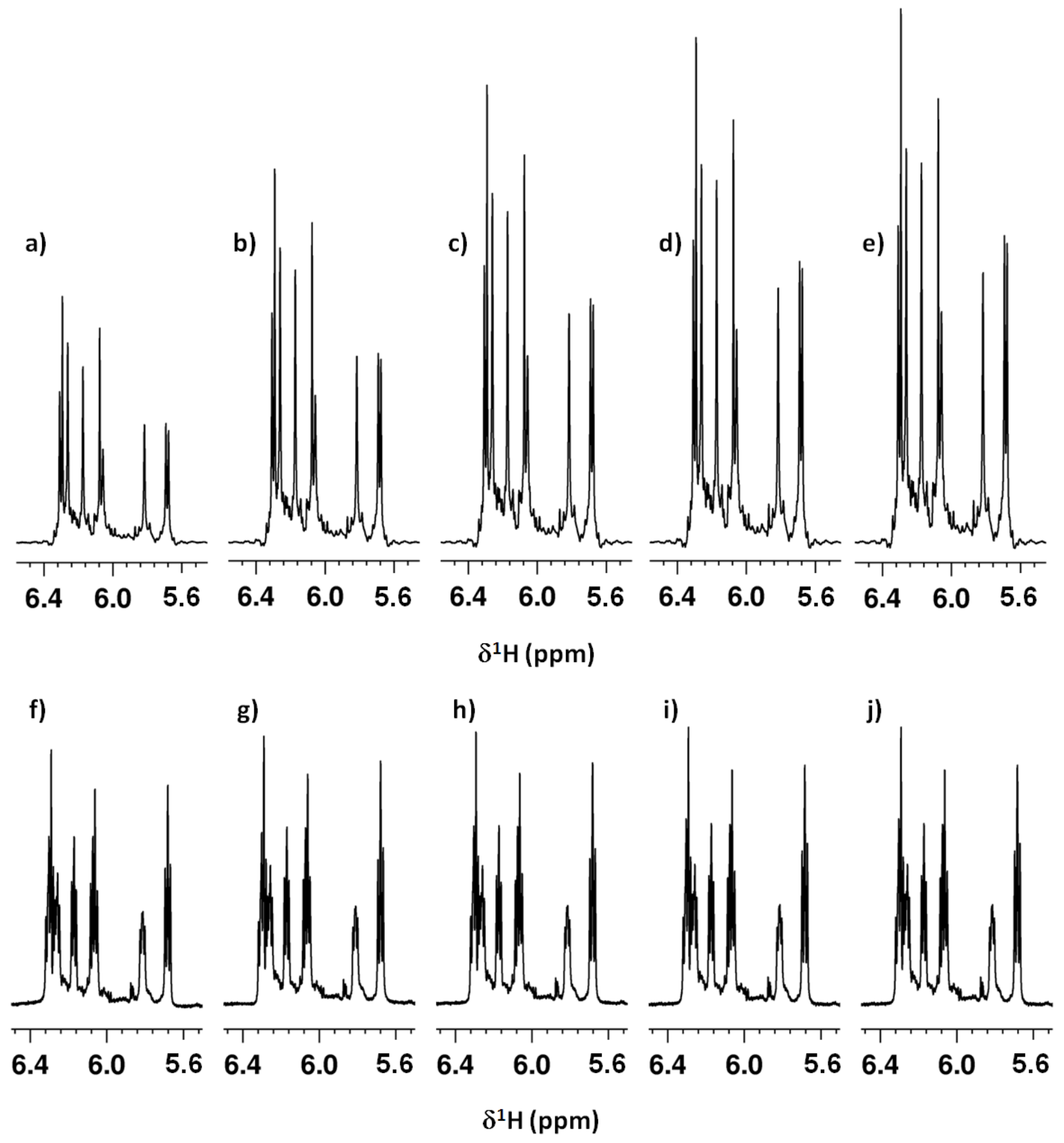

Figure S2: Effects on the appearance of the $\mathrm{H} 1^{\prime} / \mathrm{H} 5$ resonance region of the $1 \mathrm{D}{ }^{1} \mathrm{H}-\mathrm{HOBS}$ NMR spectrum (a-e) and standard 1D ${ }^{1} \mathrm{H}$ NMR spectrum (f-j) of $d(G C C T G C)$ using different

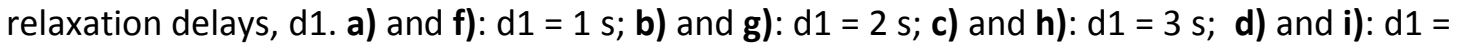
$4 \mathrm{~s} ; \mathrm{e})$ and $\mathrm{j}$ ): $\mathrm{d} 1=5 \mathrm{~s}$. For the HOBS data, the $\mathrm{H} 1^{\prime} / \mathrm{H} 5$ resonance region of the NMR spectrum was selected using a $600 \mathrm{~Hz}$ band-selective reburp $180^{\circ}$ pulse offset from the transmitter frequency by $710.5 \mathrm{~Hz}$. Presaturation of the residual $\mathrm{HOD}$ resonance was achieved at the transmitter frequency using low power irradiation during part of $\mathrm{d} 1$ in each case. The receiver gain was set to 2050 for the series a) - e) and to 228 for the series $\mathbf{f}$ ) - j). All spectra in each series are presented with the same vertical scaling factor. 


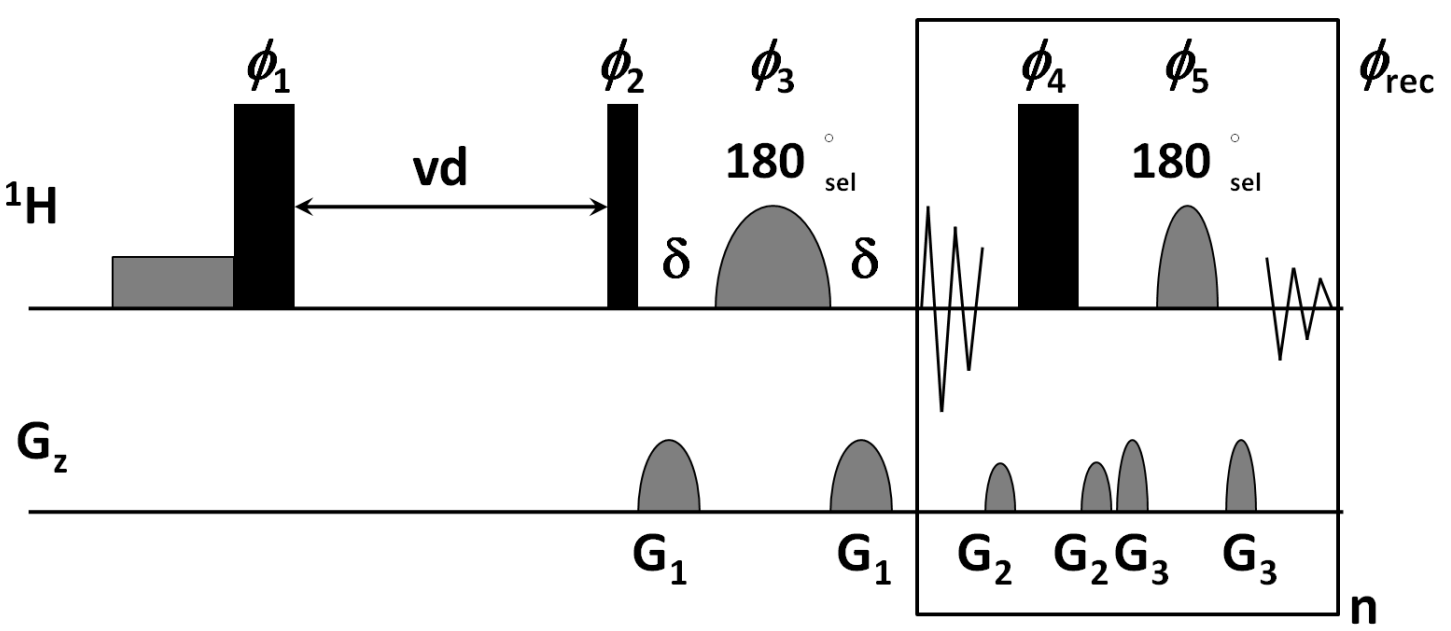

Figure S3: Pulse sequence used for acquiring $T_{1}$ inversion recovery data using a HOBS data acquisition scheme with presaturation to suppress the residual $\underline{H} O D$ signal from the aqueous sample. vd is a variable delay set according to a list of inter-pulse delay values (see main text for details). $\phi_{1}=x,-x ; \phi_{2}=\phi_{3}=x,-x,-x, x, y,-y,-y, y ; \phi_{4}=x,-x ; \phi_{5}=-x, x ; \phi_{\text {rec }}=x,-x,-x, x, y,-y,-$ $y, y ; G_{1}=60 \%, G_{2}=23 \%$ and $G_{3}=41 \%$ with a 1 ms duration ( $\delta$ ). Sine-shaped gradient pulses were used throughout. Narrow black bars correspond to hard $90^{\circ}$ pulses, wide black bars correspond to hard $180^{\circ}$ pulses and soft $180^{\circ}$ pulses are as indicated. A band-selective reburp pulse was used for selective inversion of magnetization. A low-powered pulse was applied at the solvent resonance offset during the recycle delay for suppression purposes. 


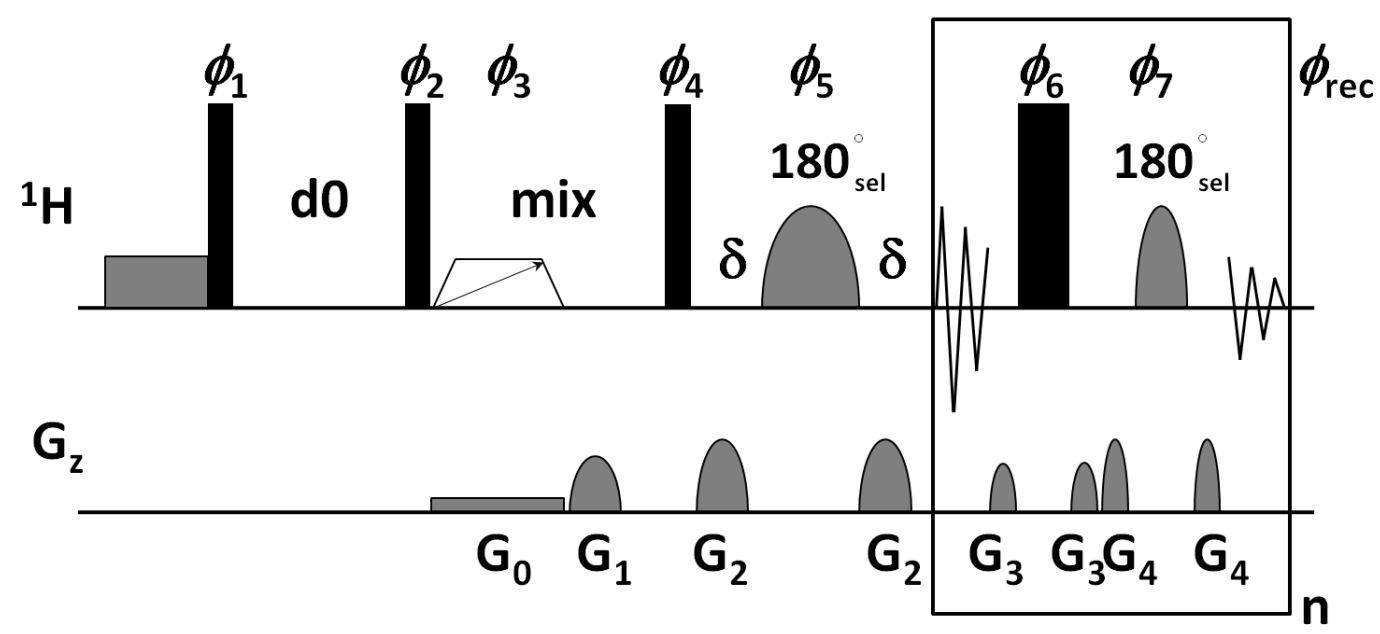

Figure S4: Pulse sequence used for acquiring zero-quantum suppressed 2D band-selective NOESY NMR spectra with homonuclear broadband proton decoupling. $\phi_{1}=x,-x ; \phi_{2}=x[8],-x[8] ; \phi_{3}$ $=x ; \phi_{4}=x, x,-x,-x, y, y,-y,-y ; \phi_{5}=x,-x,-x, x, y,-y,-y, y ; \phi_{6}=-x, x ; \phi_{7}=x,-x ; \phi_{\mathrm{rec}}=x,-x,-x, x, y,-$ $y,-y, y ; G_{0}=10 \%, G_{1}=40 \%, G_{2}=60 \%, G_{3}=23 \%$ and $G_{4}=41 \%$ with a 1 ms duration $(\delta)$. Sineshaped gradient pulses were used throughout. Narrow black bars correspond to hard $90^{\circ}$ pulses, wide black bars correspond to hard $180^{\circ}$ pulses and soft $180^{\circ}$ pulses are as indicated. A band-selective reburp pulse was used for selective inversion of magnetization. A lowpowered pulse was applied at the solvent resonance offset during the recycle delay for suppression purposes. A smoothed-CHIRP pulse of duration $20 \mathrm{~ms}$ was applied during the mixing time for ZQC suppression. 


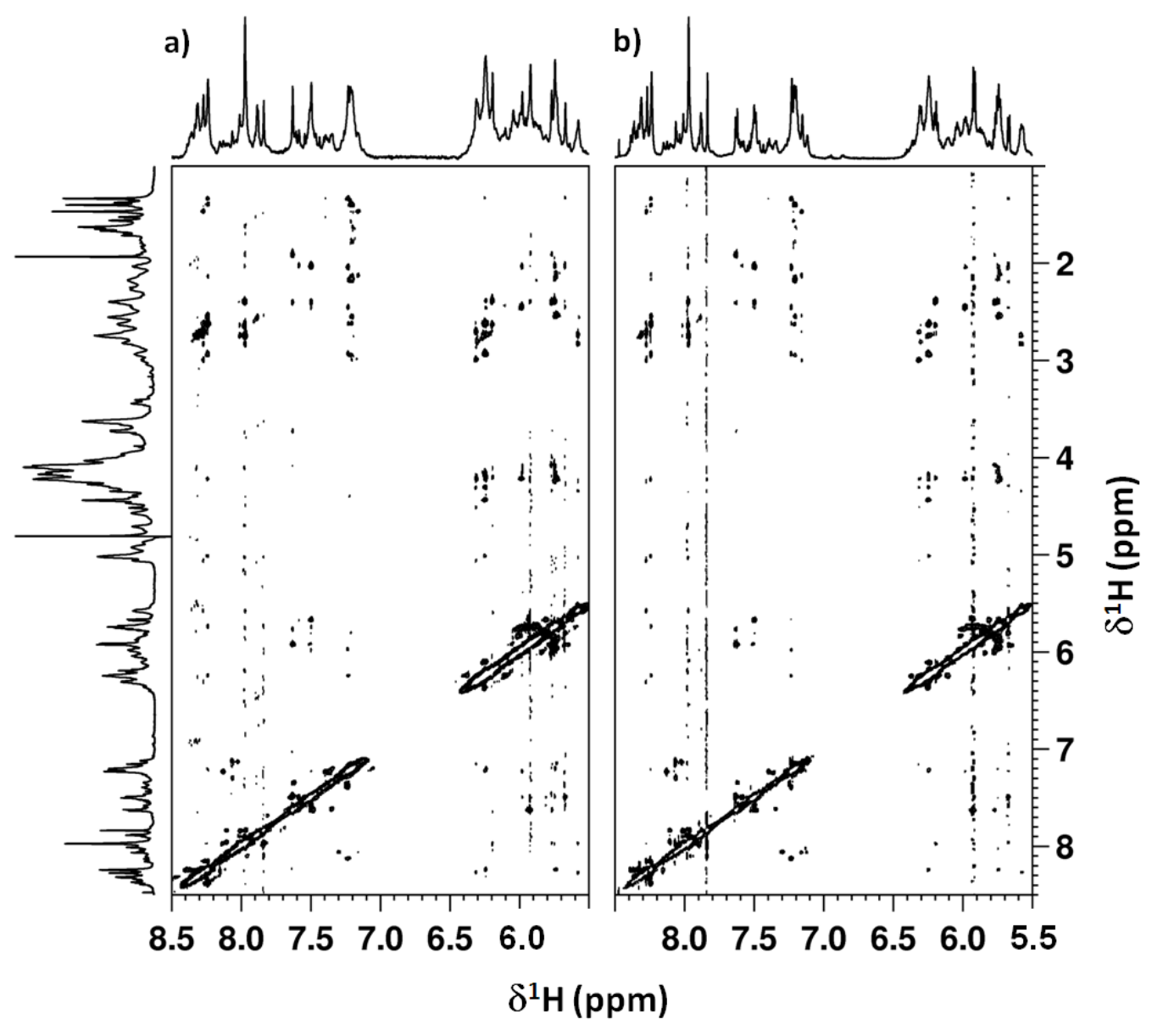

Figure S5: Combined data sets from band-selected- (a) and HOBS- (b) $2 \mathrm{D}\left[{ }^{1} \mathrm{H},{ }^{1} \mathrm{H}\right]$ NOESY for the 10mer DNA duplex d(CGATATATCG) 2 . Separate excitation regions were used for each half of each data set which were then co-added following full data processing. Band-selective reburp pulses were used to select $\mathrm{H}^{\prime} / \mathrm{H} 5$ (bandwidth $=600 \mathrm{~Hz}$ ) and aromatic/H6 (bandwidth $=990 \mathrm{~Hz}$ ) regions separately. 1D ${ }^{1} \mathrm{H}$ NMR spectra appropriate to each type of data acquisition scheme are shown as $\omega_{2}$ projections in each case. The full $1 \mathrm{D}^{1} \mathrm{H}$ NMR spectrum is shown as the $\omega_{1}$ projection. 


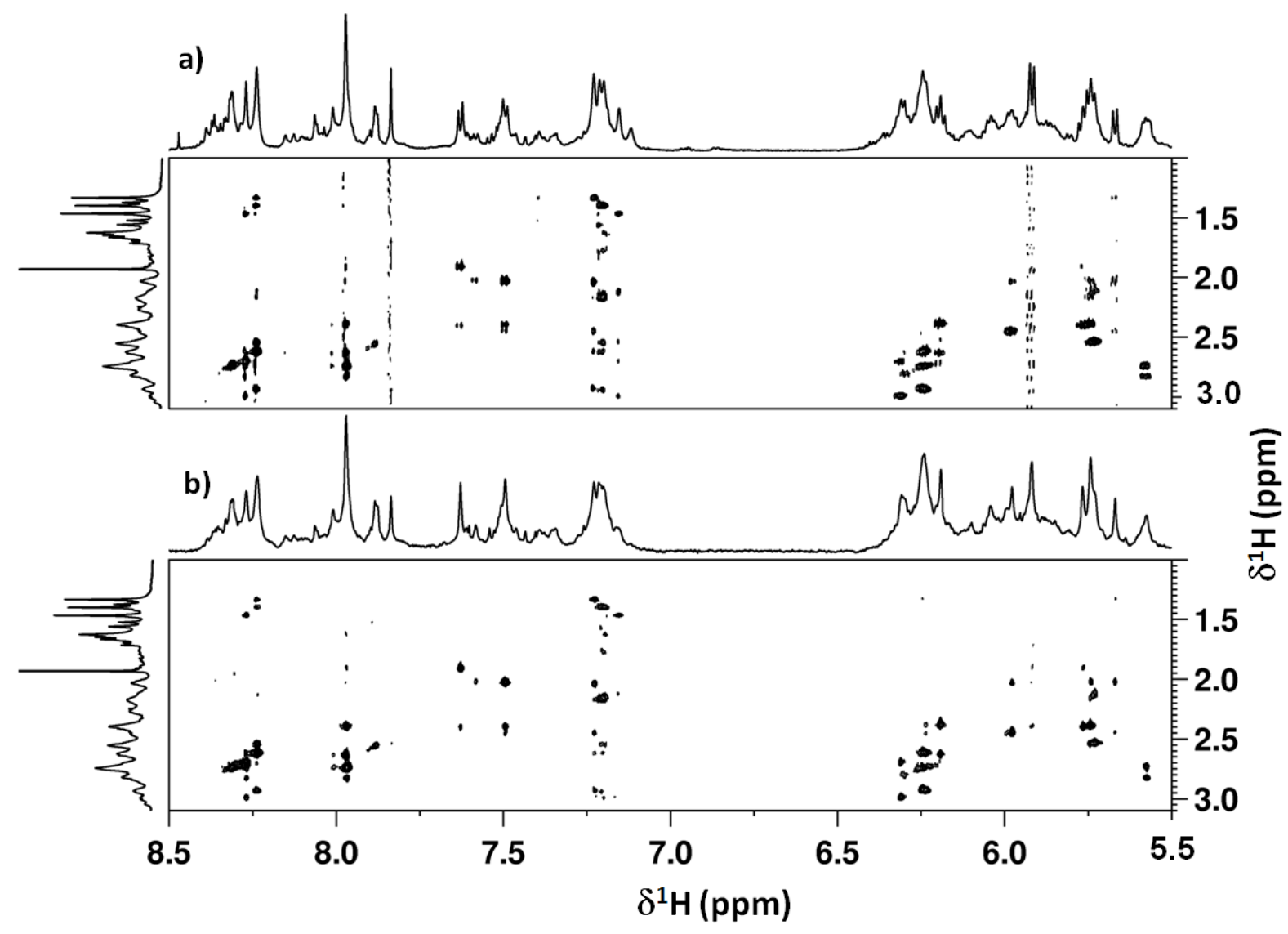

Figure S6: Expansion of the aromatic and $\mathrm{H}^{\prime} / \mathrm{H} 5$ to $\mathrm{H}^{\prime} / \mathrm{H}^{\prime \prime} / \mathrm{CH}_{3}$ cross-peak regions of combined data sets from band selective- (a) and HOBS- (b) $2 \mathrm{D}\left[{ }^{1} \mathrm{H},{ }^{1} \mathrm{H}\right]$ NOESY NMR spectra for the 10-mer DNA duplex d(CGATATATCG) ${ }_{2}$. Other features are as detailed in the caption to Figure $\mathbf{S 5 .}$ 


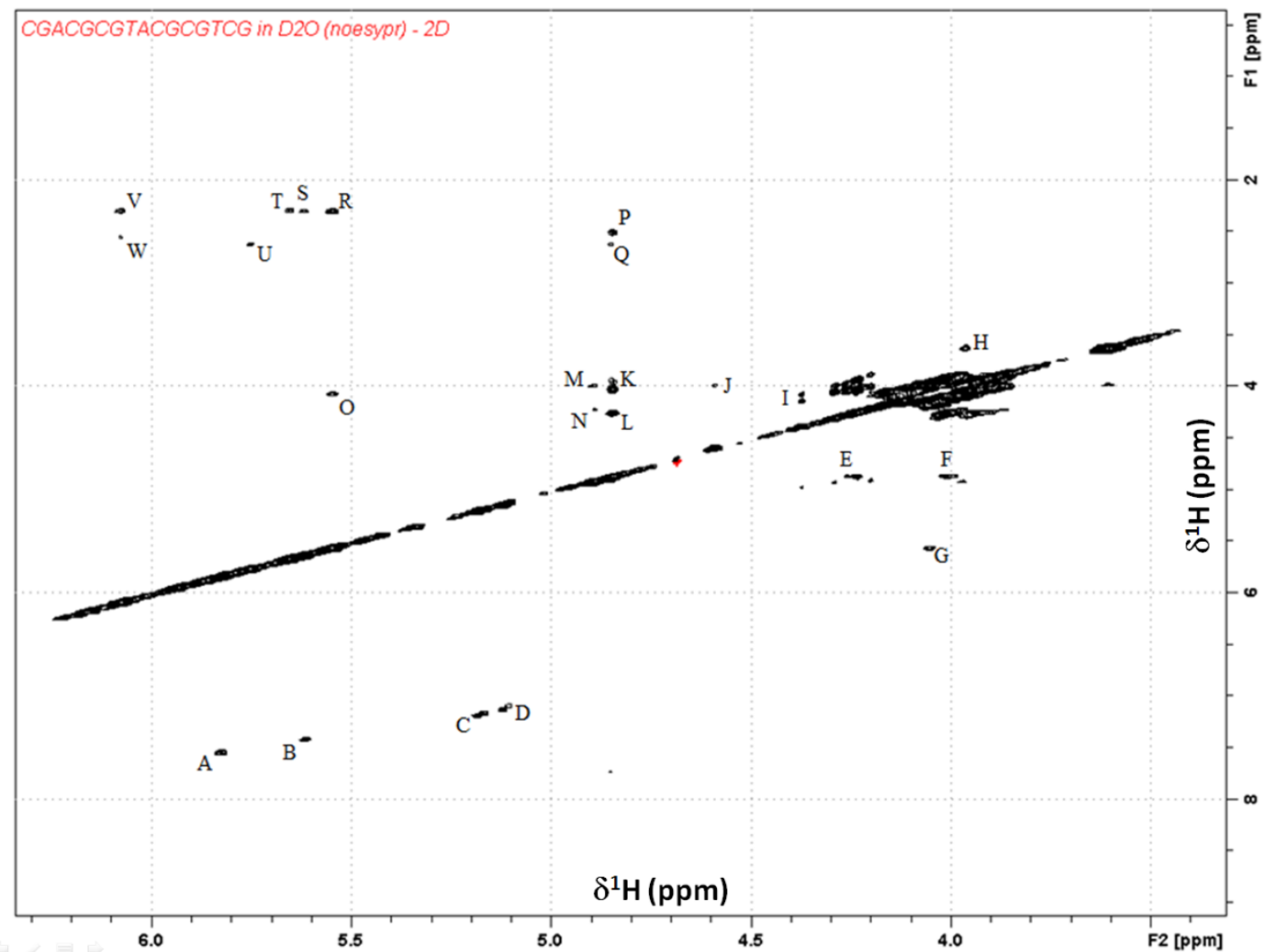

Figure S7: Strip plot in the $\mathrm{H} 1^{\prime} / \mathrm{H} 5 / \mathrm{H}^{\prime} / \mathrm{H}^{\prime} / \mathrm{H}^{\prime} / \mathrm{H} 5^{\prime \prime}$ resonance region of the $600 \mathrm{MHz} 2 \mathrm{D}\left[{ }^{1} \mathrm{H},{ }^{1} \mathrm{H}\right]$ NOESY NMR spectrum of the 16-mer DNA duplex d(CGACGCGTACGCGTCG) ${ }_{2}$ in $\mathrm{D}_{2} \mathrm{O}$ displayed with a high threshold level. Peaks used in the volume integration study described in the main text are shown labelled for ease of visualizing the reported data. 


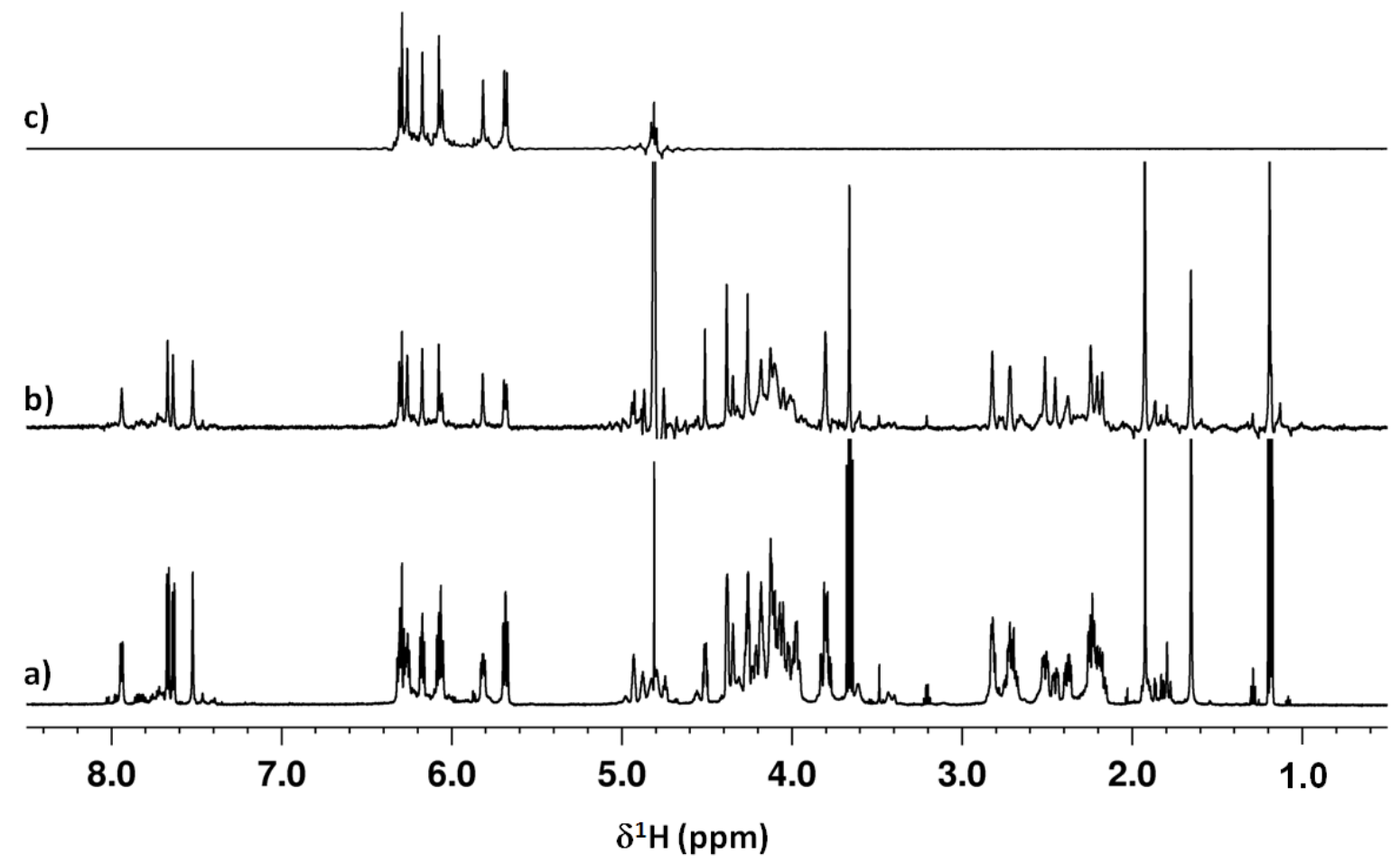

Figure 58: $600 \mathrm{MHz} 1 \mathrm{D}{ }^{1} \mathrm{H}$ NMR spectra for $\mathrm{d}(\mathrm{GCCTGC})$ in $\mathrm{D}_{2} \mathrm{O}$ using various data acquisition schemes. a) Standard $1 \mathrm{D}^{1} \mathrm{H}$ NMR spectrum using a single pulse/acquire acquisition scheme incorporating solvent presaturation - number of transients (ns) $=16$; b) Pure-shift (HOBB) 1D ${ }^{1} \mathrm{H}$ NMR spectrum with solvent presaturation - ns = 4096; c) HOBS $-1 \mathrm{D}^{1} \mathrm{H}$ NMR spectrum using a $600 \mathrm{~Hz}$ band-selective reburp pulse offset by $710.5 \mathrm{~Hz}$ from the transmitter frequency $-\mathrm{ns}=64$. No solvent presaturation was used during the acquisition of the HOBS data. 
Table S1: HOBS ${ }^{1}{ }^{H} \mathrm{~T}_{1}$ inversion-recovery relaxation data for $\mathrm{H} 5$ and $\mathrm{H}^{\prime}$ resonances from the oligonucleotide $\mathrm{d}(\mathrm{GCCTGC})$.

\begin{tabular}{|c|c|c|c|c|c|c|c|c|c|c|}
\hline \multicolumn{10}{|c|}{ H5/H1' Resonance Region } \\
\hline \multirow{2}{*}{ Peak $^{\mathbf{a}}$} & \multicolumn{9}{|c|}{$\mathbf{T}_{\mathbf{1}}$ by Integration (s) } \\
\cline { 2 - 12 } & $\mathbf{\# 1}$ & $\mathbf{\# 2}$ & $\mathbf{\# 3}$ & $\mathbf{A v g .}$ & $\mathbf{S t . ~ D e v . ~}$ & $\mathbf{\# 1}$ & $\mathbf{\# 2}$ & $\mathbf{\# 3}$ & Avg. & St. Dev. \\
\hline $\mathbf{1}$ & 1.845 & 1.852 & 1.846 & 1.848 & 0.004 & 1.673 & 1.670 & 1.665 & 1.669 & 0.004 \\
\hline $\mathbf{2}$ & 2.059 & 2.051 & 2.054 & 2.055 & 0.004 & 2.166 & 2.165 & 2.177 & 2.169 & 0.007 \\
\hline $\mathbf{3}$ & 1.742 & 1.746 & 1.747 & 1.745 & 0.003 & 1.707 & 1.699 & 1.712 & 1.706 & 0.007 \\
\hline $\mathbf{4}$ & 1.852 & 1.853 & 1.861 & 1.855 & 0.005 & 1.918 & 1.905 & 1.924 & 1.916 & 0.010 \\
\hline $\mathbf{5}$ & 1.908 & 1.910 & 1.931 & 1.916 & 0.013 & 1.852 & 1.860 & 1.857 & 1.856 & 0.004 \\
\hline $\mathbf{6}$ & 2.405 & 2.386 & 2.347 & 2.379 & 0.030 & 2.520 & 2.512 & 2.614 & 2.549 & 0.057 \\
\hline $\mathbf{7}$ & 2.114 & 2.119 & 2.125 & 2.119 & 0.006 & 1.878 & 1.885 & 1.893 & 1.885 & 0.008 \\
\hline $\mathbf{8}$ & 2.882 & 2.853 & 2.854 & 2.863 & 0.016 & 2.804 & 2.822 & 2.842 & 2.823 & 0.019 \\
\hline $\mathbf{9}$ & 2.699 & 2.751 & 2.807 & 2.752 & 0.054 & 2.986 & 2.980 & 2.976 & 2.981 & 0.005 \\
\hline
\end{tabular}

${ }^{a}$ Peak Assignments: $1-\mathrm{C}^{6} \mathrm{H}^{\prime}{ }^{\prime}, \delta^{1} \mathrm{H}=6.308$ ppm; $2-\mathrm{C}^{3} \mathrm{H}^{\prime}, \delta^{1} \mathrm{H}=6.292 \mathrm{ppm} ; 3-\mathrm{C}^{2} \mathrm{H} 1^{\prime}, \delta^{1} \mathrm{H}=6.291$ ppm; $4-\mathrm{G}^{5} \mathrm{H}^{\prime}, \delta^{1} \mathrm{H}=6.173$ ppm; $5-\mathrm{G}^{1} \mathrm{H}^{\prime}, \delta^{1} \mathrm{H}=6.075$ ppm; $6-\mathrm{C}^{3} \mathrm{H} 5, \delta^{1} \mathrm{H}=6.057$ ppm; $7-$ $\mathrm{T}^{4} \mathrm{H} 1^{\prime}, \delta^{1} \mathrm{H}=5.816 \mathrm{ppm} ; 8-\mathrm{C}^{6} \mathrm{H} 5, \delta^{1} \mathrm{H}=5.691 \mathrm{ppm} ; 9-\mathrm{C}^{2} \mathrm{H} 5, \delta^{1} \mathrm{H}=5.675 \mathrm{ppm}$. Measurements are reported from experiments carried out in triplicate.

Table S2: Standard- ${ }^{1} \mathrm{H} \mathrm{T}_{1}$ inversion-recovery relaxation data for $\mathrm{H} 5$ and $\mathrm{H}^{\prime}$ ' resonances from the oligonucleotide d(GCCTGC).

\begin{tabular}{|c|c|c|c|c|c|c|c|c|c|c|}
\hline \multicolumn{11}{|c|}{ H5/H1' Resonance Region } \\
\hline \multirow[t]{2}{*}{ Peak $^{a}$} & \multicolumn{5}{|c|}{$\mathrm{T}_{1}$ by Integration (s) } & \multicolumn{5}{|c|}{$\mathrm{T}_{1}$ by Peak Picking (s) } \\
\hline & $\# 1$ & $\# 2$ & $\# 3$ & Avg. & St. Dev. & $\# 1$ & $\# 2$ & $\# 3$ & Avg. & St. Dev. \\
\hline 1 & --- & --- & --- & --- & --- & 1.849 & 1.826 & 1.819 & 1.831 & 0.016 \\
\hline 2 & --- & --- & --- & --- & --- & 2.072 & 2.060 & 2.051 & 2.061 & 0.011 \\
\hline 3 & --- & --- & --- & --- & --- & 1.725 & 1.702 & 1.686 & 1.704 & 0.020 \\
\hline 4 & 1.864 & 1.842 & 1.845 & 1.850 & 0.012 & 1.872 & 1.848 & 1.841 & 1.854 & 0.016 \\
\hline 5 & --- & --- & --- & --- & --- & 1.912 & 1.879 & 1.863 & 1.885 & 0.025 \\
\hline 6 & --- & --- & --- & --- & --- & 2.543 & 2.505 & 2.464 & 2.504 & 0.040 \\
\hline 7 & 2.048 & 2.043 & 2.058 & 2.050 & 0.008 & 2.007 & 2.005 & 2.001 & 2.004 & 0.003 \\
\hline 8 & --- & --- & --- & --- & --- & 2.870 & 2.817 & 2.780 & 2.822 & 0.045 \\
\hline 9 & --- & --- & --- & --- & --- & 2.779 & 2.774 & 2.711 & 2.755 & 0.038 \\
\hline
\end{tabular}

${ }^{a}$ Peak Assignments: $1-\mathrm{C}^{6} \mathrm{H}^{\prime}, \delta^{1} \mathrm{H}=6.308$ ppm; $2-\mathrm{C}^{3} \mathrm{H}^{\prime}, \delta^{1} \mathrm{H}=6.292 \mathrm{ppm} ; 3-\mathrm{C}^{2} \mathrm{H} 1^{\prime}, \delta^{1} \mathrm{H}=6.291$ ppm; $4-\mathrm{G}^{5} \mathrm{H}^{\prime}, \delta^{1} \mathrm{H}=6.173$ ppm; $5-\mathrm{G}^{1} \mathrm{H}^{\prime}, \delta^{1} \mathrm{H}=6.075$ ppm; $6-\mathrm{C}^{3} \mathrm{H} 5, \delta^{1} \mathrm{H}=6.057$ ppm; $7-$ $\mathrm{T}^{4} \mathrm{H} 1^{\prime}, \delta^{1} \mathrm{H}=5.816$ ppm; $8-\mathrm{C}^{6} \mathrm{H} 5, \delta^{1} \mathrm{H}=5.691 \mathrm{ppm} ; 9-\mathrm{C}^{2} \mathrm{H} 5, \delta^{1} \mathrm{H}=5.675 \mathrm{ppm}$. Measurements are reported from experiments carried out in triplicate. Integration was carried out only for those peaks that were clearly resolved. 
Table S3: $\mathrm{HOBS}-{ }^{1} \mathrm{HT}_{1}$ inversion-recovery relaxation data for aromatic resonances from the oligonucleotide $\mathrm{d}(\mathrm{GCCTGC})$.

\begin{tabular}{|c|c|c|c|c|c|c|c|c|c|c|}
\hline \multicolumn{10}{|c|}{ Aromatic Resonance Region } \\
\hline \multirow{2}{*}{ Peaks $^{\text {a }}$} & \multicolumn{9}{|c|}{$\mathbf{T}_{\mathbf{1}}$ by Integration (s) } \\
\cline { 2 - 11 } & $\mathbf{\# 1}$ & $\mathbf{\# 2}$ & $\mathbf{\# 3}$ & Avg. & St. Dev. & $\# \mathbf{1}$ & $\mathbf{\# 2}$ & $\mathbf{\# 3}$ & Avg. & St. Dev. \\
\hline $\mathbf{1}$ & 1.658 & 1.663 & 1.642 & 1.654 & 0.011 & 1.384 & 1.390 & 1.395 & 1.390 & 0.006 \\
\hline $\mathbf{2}$ & 1.382 & 1.381 & 1.384 & 1.382 & 0.002 & 1.384 & 1.406 & 1.395 & 1.395 & 0.011 \\
\hline $\mathbf{3}$ & 1.589 & 1.583 & 1.577 & 1.583 & 0.006 & 1.624 & 1.639 & 1.629 & 1.631 & 0.008 \\
\hline $\mathbf{4}$ & 1.587 & 1.582 & 1.581 & 1.583 & 0.003 & 1.631 & 1.637 & 1.629 & 1.632 & 0.004 \\
\hline
\end{tabular}

${ }^{a}$ Peak Assignments: $1-\mathrm{C}^{3} \mathrm{H} 6, \delta^{1} \mathrm{H}=7.940$ ppm; $2-\mathrm{C}^{2} \mathrm{H} 6, \delta^{1} \mathrm{H}=7.670$ ppm; $3-\mathrm{C}^{6} \mathrm{H} 6, \delta^{1} \mathrm{H}=7.630$ ppm; $4-\mathrm{T}^{4} \mathrm{H} 6, \delta^{1} \mathrm{H}=7.520 \mathrm{ppm}$. Measurements are reported from experiments carried out in triplicate.

Table S4: Standard- ${ }^{1} \mathrm{H} \mathrm{T}_{1}$ inversion-recovery relaxation data for aromatic resonances from the oligonucleotide $\mathrm{d}(\mathrm{GCCTGC})$.

\begin{tabular}{|c|c|c|c|c|c|c|c|c|c|c|}
\hline \multicolumn{11}{|c|}{ Aromatic Resonance Region } \\
\hline \multirow[t]{2}{*}{ Peaks $^{a}$} & \multicolumn{5}{|c|}{$T_{1}$ by Integration (s) } & \multicolumn{5}{|c|}{$\mathrm{T}_{1}$ by Peak Picking (s) } \\
\hline & $\# 1$ & $\# 2$ & $\# 3$ & Avg. & St. Dev. & $\# 1$ & $\# 2$ & $\# 3$ & Avg. & St. Dev. \\
\hline 1 & 1.609 & 1.599 & 1.601 & 1.603 & 0.005 & 1.605 & 1.585 & 1.566 & 1.585 & 0.020 \\
\hline 2 & 1.396 & 1.382 & 1.382 & 1.387 & 0.008 & 1.404 & 1.384 & 1.377 & 1.388 & 0.014 \\
\hline 3 & 1.627 & 1.607 & 1.611 & 1.615 & 0.011 & 1.642 & 1.605 & 1.589 & 1.612 & 0.027 \\
\hline 4 & 1.566 & 1.561 & 1.565 & 1.564 & 0.003 & 1.597 & 1.583 & 1.584 & 1.588 & 0.008 \\
\hline
\end{tabular}

${ }^{a}$ Peak Assignments: $1-\mathrm{C}^{3} \mathrm{H} 6, \delta^{1} \mathrm{H}=7.940$ ppm; $2-\mathrm{C}^{2} \mathrm{H} 6, \delta^{1} \mathrm{H}=7.670$ ppm; $3-\mathrm{C}^{6} \mathrm{H} 6, \delta^{1} \mathrm{H}=7.630$ ppm; $4-\mathrm{T}^{4} \mathrm{H} 6, \delta^{1} \mathrm{H}=7.520 \mathrm{ppm}$. Measurements are reported from experiments carried out in triplicate. 